\title{
The challenges of sodium measurements: indirect versus direct ion-selective method
}

\author{
Julie Refardt ${ }^{1,2, *}$, Clara Odilia Sailer ${ }^{1,2, *}$, Irina Chifu ${ }^{3}$, Bettina Winzeler ${ }^{1,2}$, Ingeborg Schnyder, \\ Martin Fassnacht ${ }^{3,4}$, Wiebke Fenske ${ }^{5,6}$ and Mirjam Christ-Crain ${ }^{1,2}$ on behalf of the CODDI-Investigators
}

${ }^{1}$ Departments of Endocrinology, Diabetology and Metabolism, University Hospital Basel, Basel, Switzerland, ${ }^{2}$ Department of Clinical Research, University of Basel, Basel, Switzerland, ${ }^{3}$ Division of Endocrinology and Diabetes, Department of Internal Medicine I, University Hospital, University of Würzburg, Würzburg, Germany, ${ }^{4}$ Central Laboratory, University Hospital Würzburg, Würzburg, Germany, ${ }^{5}$ Department of Endocrinology and Nephrology, University of Leipzig, Leipzig, Germany, and ${ }^{6}$ Leipzig University Medical Center, IFB AdiposityDiseases, Leipzig, Germany

\begin{abstract}
Background: Diagnosis and treatment of dysnatremia is challenging and further complicated by the pitfalls of different sodium measurement methods. Routinely used sodium measurements are the indirect (plasma/serum) and direct (whole blood) ion-selective electrode (ISE) method, showing discrepant results especially in the setting of acute illness. Few clinicians are aware of the differences between the methods in clinically stable patients or healthy volunteers. Methods: Data of 140 patients and 91 healthy volunteers undergoing osmotic stimulation with hypertonic saline infusion were analyzed. Sodium levels were measured simultaneously by indirect and direct ISE method before and at different time points during osmotic stimulation up to a sodium threshold of $\geq 150 \mathrm{mmol} / \mathrm{L}$. The primary outcome was the difference in sodium levels between the indirect and direct ISE method.

Results: 878 sodium measurements were analyzed. Mean (S.D.) sodium levels ranged from $141 \mathrm{mmol} / \mathrm{L}$ (2.9) to $151 \mathrm{mmol} / \mathrm{L} \mathrm{(2.1)} \mathrm{by} \mathrm{the} \mathrm{indirect} \mathrm{ISE} \mathrm{compared} \mathrm{to} 140 \mathrm{mmol} / \mathrm{L} \mathrm{(3)} \mathrm{to} 149 \mathrm{mmol} / \mathrm{L}$ (2.8) by the direct ISE method. The interclass correlation coefficient between the two methods was 0.844 ( $95 \% \mathrm{Cl}: 0.823-0.863$ ). On average, measurements by the indirect ISE were $1.9 \mathrm{mmol} / \mathrm{L}$ (95\% Cl limits: -3.2 to 6.9) higher than those by the direct ISE method $(P<0.001)$. The tendency of the indirect ISE method resulting in higher levels increased with increasing sodium levels.

Conclusion: Intra-individual sodium levels differ significantly between the indirect and direct ISE method also in the absence of acute illness. It is therefore crucial to adhere to the same method in critical situations to avoid false decisions due to measurement differences.
\end{abstract}

\section{Introduction}

Hypo- and hypernatremia are the most common electrolyte disorders $(1,2)$. Precise and reliable sodium measurements are crucial, as incorrect treatment can lead to lifethreatening complications $(3,4,5)$. Accordingly, current guidelines suggest strict treatment limits of $8-10 \mathrm{mmol} / \mathrm{L}$ per $24 \mathrm{~h}$ when treating chronic dysnatremia (4).

Today, most laboratories use the indirect ion-selective electrode (ISE) method to measure sodium levels $(6,7,8)$.
European Journal of
The indirect ISE method requires a predilution step to expand the measurable concentration range and has become the standard analysis in most hospitals. Conversely, the direct ISE method requires a whole blood sample (e.g., blood gas analysis) and is usually used in the emergency or intensive care unit due to its rapid results (8).

Over the last years, several studies showed a discrepancy in sodium levels between the indirect and

Published by Bioscientifica Ltd. 
direct ISE method $(9,10,11,12)$, especially in patients presenting at the emergency department (9), at the intensive care unit $(13,14)$ or in patients hospitalized due to other reasons (10). The main reason for this discrepancy is reported to be caused by the displacement of plasma water due to abnormal protein and lipid levels, affecting the dilution concentration in the indirect ISE method and therefore resulting in 'pseudohyponatremia' or 'pseudohypernatremia' (13, 14, 15). Accordingly, most authors agree that the direct ISE method should be preferred in patients with abnormal protein and lipid concentrations and that for routine sodium measurements, a correction for the estimated water content should be used $(11,13)$.

In other settings than described above, most clinicians consider the two methods to be interchangeable and awareness of a potential sodium measurement discrepancy is lacking. This becomes important in the treatment of hyponatremia or in case of patients requiring hypertonic saline infusion as a diagnostic test for the polyuria polydipsia syndrome $(16,17)$. Both measurement methods are often used in these situations and decisions are based on sodium changes of a few mmol/L only. The same applies to the treatment decisions in hypernatremia.

To investigate the sodium measurement method precision, we analyzed repetitive simultaneous sodium measurements by the indirect and direct ISE method of stable patients and healthy volunteers undergoing osmotic stimulation with hypertonic saline infusion.

\section{Methods}

\section{Study design and participants}

One hundred forty-one patients with either primary polydipsia or central diabetes insipidus from the prospective multicenter CODDI study (17) and of 91 healthy volunteers form the CoNORM study (18) undergoing the same protocol of osmotic stimulation with hypertonic (3\%) saline infusion were included from 2013 to 2017. Full details of the studies rationales, designs and statistical analyses have been published elsewhere $(17,18)$. Both studies were registered on ClinicalTrials.gov (NCT01940614/NCT02647736). They were approved by the Ethical Committee Northwest and Central Switzerland, University of Basel, Basel, Switzerland and the Ethical Committee of the University of Würzburg, Würzburg, Germany. Written informed consent has been obtained from each patient or participant after full explanation of the purpose and nature of all procedures used.
Eligible patients were of age 18 years or older and had confirmed primary polydipsia or central diabetes insipidus. Patients with glucosuric polyuria, electrolyte disorders, untreated or insufficiently replaced pituitary-, adrenalor thyroid deficiency or impaired kidney function, heart failure, uncontrolled hypertension or a history of epilepsy were ineligible.

Healthy volunteers were of age 18 years or older and were normonatremic. Exclusion criteria included a history or presence of polyuria polydipsia syndrome (which comprises all forms of diabetes insipidus and primary polydipsia), any chronic or therapy-requiring diseases, chronic alcohol consumption or drug intake (except oral contraception).

Pregnancy or breastfeeding was an exclusion criterion in both studies.

\section{Test protocol}

At baseline, a detailed medical history and standardized clinical and biochemical evaluation was obtained. No food intake was allowed after midnight, no fluid intake after 6:00 am on the test day. Diuretic or antidiuretic medications were discontinued for at least $24 \mathrm{~h}$, and smoking and alcohol were prohibited for at least $12 \mathrm{~h}$ before the test. Participants then underwent the hypertonic saline infusion test between 8:00 am and 11:00 am After an initial $250 \mathrm{~mL}$ bolus, peripheral intravenous infusion of $3 \%$ saline was continued at an infusion rate of $0.15 \mathrm{~mL}$ per $\mathrm{kg}$ bodyweight per minute. Sodium levels were controlled every $30 \mathrm{~min}$ by routine laboratory indirect and direct ISE method until sodium levels reached $\geq 150 \mathrm{mmol} / \mathrm{L}$. Once this level was reached, sodium levels were immediately re-lowered by oral hydration and dextrose infusion (recovery period), with sodium measurements using both methods available for healthy volunteers.

\section{Laboratory measurements}

Blood samples were immediately transported to the central laboratory in standard biochemistry tubes. Lithium-heparin plasma tubes were centrifuged, and plasma sodium levels were then analyzed by indirect ISE method (University Hospital Basel and Würzburg: cobas ${ }^{\circledR} 8000$ modular analyzer, Roche Diagnostics). Heparinized blood gas tubes were used to collect venous blood gas samples, sodium levels were measured by direct ISE method (University Hospital Basel: ABL 800 Flex®, Radiometer GmbH; University Hospital Würzburg: ABL90 FLEX®, Radiometer $\mathrm{GmbH}$ ). The coefficient of variation 
at the University Hospital Basel was $1.99 \%$ for the indirect and $0.42 \%$ for the direct ISE method. For the University Hospital Würzburg the coefficient of variation was $1.14 \%$ for the indirect and $0.52 \%$ for the direct ISE method. Plasma osmolality was measured in lithium-heparin plasma tubes using the freezing point method.

\section{Statistical analysis}

One patient with central diabetes insipidus was excluded from analysis due to missing simultaneous measurements. The full analysis set included all data where sodium was measured simultaneously with the indirect and direct ISE method ( $n=878)$. Results are shown as mean and standard deviation (s.D.) with range (min, max) and number and percentage (\%) appropriately.

The primary outcome was to assess the agreement between the indirect and direct ISE method using the interclass correlation coefficient (ICC). The ICC is a type of correlation used to assess the consistency of two observers, in our case the indirect and direct ISE method, measuring the same quantity, in our case sodium levels. The $t$-test was applied for two group comparisons of normally distributed continuous data. A Bland-Altman Plot was created for agreement analysis. Measurement differences outside of the 95\% CI limits of the BlandAltman Plot were analyzed to identify potential predisposing factors for mismatch such as age, sex, BMI and diagnosis.

To visually analyze the time course of the measurement method difference, nine random patients with sodium measurements at all time points were selected and plotted.

To assess the differences between different study sites, the correlation as well as the mean difference between the University Hospital Basel and the University Hospital Würzburg were compared.

Statistical analyses were performed using the statistic programs GraphPad PRISM version 7.03 and R Statistical Software (19). Hypothesis testing was two sided and $P$ values $<0.05$ were considered statistically significant.

\section{Results}

Sodium measurements of 140 patients (82 patients with primary polydipsia, 58 patients with central diabetes insipidus) and 91 healthy volunteers were analyzed (Table 1). All participants were in a stable condition and had blood glucose and albumin levels within the reference range. Hyperlipidemia was also excluded in the patient group. In total, 878 sodium measurements ranging from 132 to $161 \mathrm{mmol} / \mathrm{L}$ were analyzed.

At baseline, mean (s.D.) sodium value was $141 \mathrm{mmol} / \mathrm{L}$ (2.9) by the indirect ISE compared to $140 \mathrm{mmol} / \mathrm{L}$ (3) by the direct ISE method (estimated mean difference of $1.54 \mathrm{mmol} / \mathrm{L}, P<0.001)$. The maximally stimulated mean (s.D.) sodium value was $151 \mathrm{mmol} / \mathrm{L}$ (2.1) by the indirect ISE and $149 \mathrm{mmol} / \mathrm{L}$ (2.8) by the direct ISE method (estimated mean difference of $3 \mathrm{mmol} / \mathrm{L}, P<0.001)$. Mean sodium value of the recovery period (healthy volunteers only) was $146 \mathrm{mmol} / \mathrm{L}$ (s.D. 4.1) for the indirect and $142 \mathrm{mmol} / \mathrm{L}$ (s.D. 3.2) for the direct measurement method (estimated mean difference of $2.2 \mathrm{mmol} / \mathrm{L}, P<0.001)$. Overall, mean sodium value was $147 \mathrm{mmol} / \mathrm{L}$ (4.8) by the indirect ISE and $145 \mathrm{mmol} / \mathrm{L}$ (4.6) by the direct ISE method (estimated mean difference of $1.9 \mathrm{mmol} / \mathrm{L}, P$ value $<0.001)$. The values at the different time points are displayed in Fig. 1 and Table 1.

Table 1 Sodium measurements of all participants during the hypertonic saline infusion test. Values shown as mean (s.D.; minimum-maximum) of the population. $P$ values refer to differences between indirect and direct ion-selective electrode measurement by paired $t$-test.

\begin{tabular}{l}
\hline \\
\hline Patients \\
Baseline $(n=138)$ \\
Stimulated $(n=192)$ \\
Healthy volunteers \\
Baseline $(n=46)$ \\
Stimulated $(n=100)$ \\
Recovery period $(n=99)$ \\
All \\
Baseline $(n=184)$ \\
Stimulated $(n=292)$
\end{tabular}

\begin{tabular}{c}
\hline Indirect ISE \\
\hline $141(2.8 ; 133-151)$ \\
$152(2.3 ; 149-161)$ \\
$140(2.1 ; 136-146)$ \\
$151(1.6 ; 149-155)$ \\
$145(3.6 ; 139-155)$ \\
$141(2.9 ; 133-151)$ \\
$151(2.1 ; 149-161)$ \\
\hline
\end{tabular}

\begin{tabular}{c}
\hline Direct ISE \\
\hline $139(3.0 ; 132-149)$ \\
$149(2.9 ; 142-161)$ \\
$138(1.7 ; 133-142)$ \\
$149(2.6 ; 137-153)$ \\
$142(3.3 ; 136-153)$ \\
$140(3.0 ; 132-149)$ \\
$149(2.8 ; 137-161)$ \\
\hline
\end{tabular}

\begin{tabular}{c}
\hline P-Osmolality \\
\hline $289(9.1 ; 265-324)$ \\
$306(9.5 ; 283-341)$ \\
$293(6.7 ; 280-306)$ \\
$313(6.6 ; 297-329)$ \\
$303(7.9 ; 286-329)$ \\
$289(8.8 ; 265-324)$ \\
$308(9.0 ; 283-341)$ \\
\hline
\end{tabular}

\begin{tabular}{l} 
P value \\
\hline$<0.001$ \\
$<0.001$ \\
$<0.001$ \\
$<0.001$ \\
$<0.001$ \\
$<0.001$ \\
$<0.001$
\end{tabular}

Baseline, baseline measurement; ISE, ion-selective electrode method; $n$, number of measurements; P, plasma; Stimulated, maximally stimulated sodium measurement by hypertonic saline infusion. 


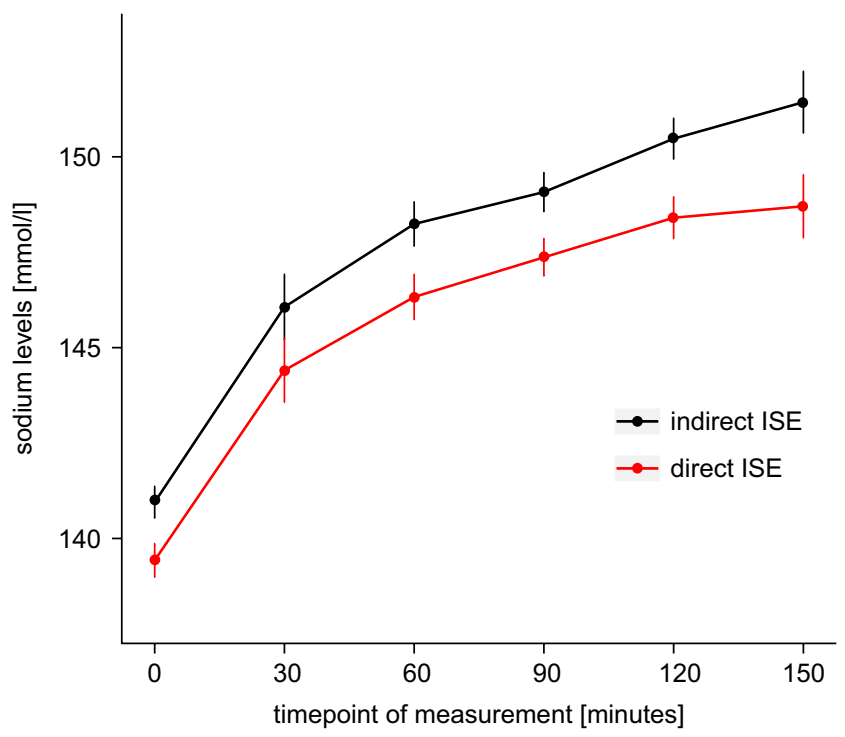

\section{Figure 1}

Course of sodium levels during the hypertonic saline infusion test according to the indirect and direct ISE measurement methods. Mean and error bar are represented at different time points.

The ICC between the indirect and direct ISE method overall was 0.86 (95\% CI $0.84-0.88$ ), for patients 0.85 (95\% CI 0.82-0.87) and for healthy volunteers 0.88 (95\% CI 0.85-0.9) (Fig. 2). The Bland-Altman Plot visualizes the agreement between the two assays (Fig. 3), indicating the mean difference of $1.9 \mathrm{mmol} / \mathrm{L}$ (95\% CI limits: lower limit -3.2; upper limit 6.9). As measurement differences ranged from -7 to $+14 \mathrm{mmol} / \mathrm{L}$, sub-analysis of all participants exceeding the 95\% CI limits of the mean difference was performed. Five healthy volunteers (80\% female) and six patients ( $67 \%$ female, $67 \%$ partial central diabetes insipidus) had measurements above the $95 \%$ CI limit, while one male healthy volunteer and four female patients ( $75 \%$ primary polydipsia) had measurements below the $95 \%$ CI limit. No predisposing factors were observed evaluating the variables age and BMI (data not shown).

The tendency of the measurement method difference (e.g. indirect ISE method being higher or lower than direct ISE method) was consistent during the course of the test; however, the extent of the difference was highly variable (Fig. 4).

Sodium measurements between the two main study centers University Hospital Basel and University Hospital Würzburg were similar, showing a correlation of $R=0.89$ in Basel and $R=0.84$ in Würzburg between the indirect and direct ISE method overall. The variation of sodium

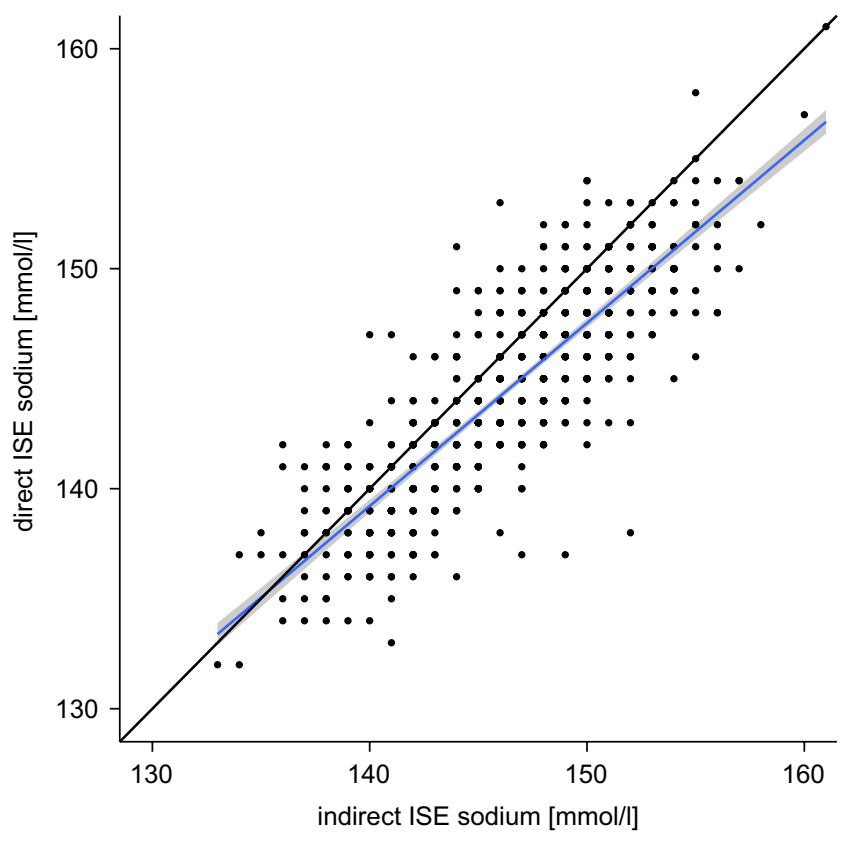

Figure 2

Correlation between indirect and direct ion selective electrode method. The blue line represents the linear regression line between the two measurements. The black line represents the ideal correlation line. A full colour version of this figure is available at https://doi.org/10.1530/EJE-19-0101.

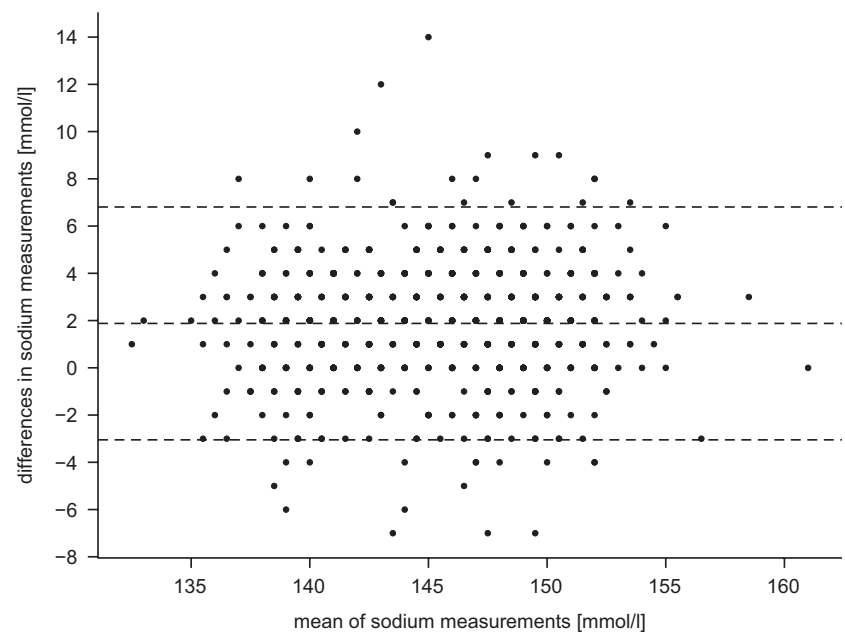

\section{Figure 3}

Bland-Altman graph of difference in sodium measurements between the indirect and the direction selective electrode method. The middle line represents the mean difference of $1.9 \mathrm{mmol} / \mathrm{L}$ between the two methods. The two outer dotted lines represent the $95 \%$ confident interval limits of agreement (lower limit -3.2, upper limit 6.9). 


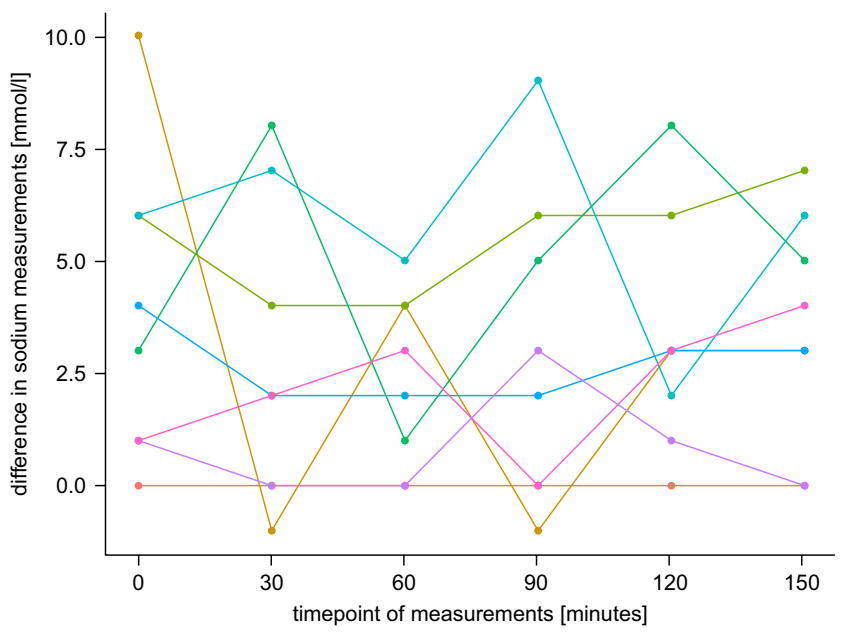

Figure 4

Difference in sodium levels between the indirect and direction selective electrode method according to nine randomly selected individual participants with at least six consecutive simultaneous sodium measurements. Every color represents one participant. Values above 0 represent sodium levels measured by indirect ISE being higher compared to the direct ISE method; values below 0 represent sodium levels measured by indirect ISE being lower compared to the direct ISE method.

measurement differences was also similar between the two centers (University Hospital Basel of $2 \mathrm{mmol} / \mathrm{L}$; University Hospital Würzburg of $2.5 \mathrm{mmol} / \mathrm{L}$ ) (Supplementary Figs $1 \mathrm{~A}, \mathrm{~B}$ and 2 , see section on supplementary data given at the end of this article).

\section{Discussion}

We here show that sodium levels differ significantly between the indirect and direct ISE method in clinically stable patients and healthy volunteers. This difference increases over repetitive measurements from the normoto the hypernatremic range, showing consistent higher levels for the indirect ISE method. The mean difference was $1.9 \mathrm{mmol} / \mathrm{L}$; however, the range of discrepancies reached from $-7 \mathrm{mmol} / \mathrm{L}$ to $+14 \mathrm{mmol} / \mathrm{L}$. A similar observation was shown in the study of Solak (9) in patients presenting at the emergency department of a tertiary care hospital, where the differences in hypernatremic sodium measurements ranged from $-18 \mathrm{mmol} / \mathrm{L}$ to $+23 \mathrm{mmol} / \mathrm{L}$ between indirect and direct ISE method.

From a laboratory perspective one could argue that a mean difference in sodium values of $1.9 \mathrm{mmol} / \mathrm{L}$ between the two different ISE methods is within an expected and acceptable range. However, from a clinician's perspective, this discrepancy cannot be disregarded. This is especially crucial in situations with chronic hypernatremia, as here similar sodium correction rates as in chronic hyponatremia $(8-10 \mathrm{mmol} / 24 \mathrm{~h}(16,20)$ or $8 \mathrm{mmol} / 24 \mathrm{~h}$ in patients with a high risk for osmotic demyelination $(21,22)$ ) are recommended (23). Incorrect treatment decisions due to variable sodium measurement methods could have fatal consequences. Furthermore, in the differential diagnosis of the polyuria polydipsia syndrome with hypertonic saline-stimulated copeptin being a new diagnostic approach (17), clinicians must be aware of the sodium measurement variances and differences.

In 20 measurements, the difference between the two methods exceeded the 95\% CI limits of the Bland-Altman Plot. Interestingly, the majority of these discrepancies occurred in female participants (75\%). An explanation for this finding could be the influence of sex hormones on body fluid and sodium regulation in women as described by Stachenfeld et al., making women potentially more susceptible for sodium measurement discrepancies $(24,25)$. Of the ten patients with large measurement differences, patients with primary polydipsia tended to have lower indirect measurements compared to the direct method, while the contrary was observed for patients with central diabetes insipidus. This could be due to the difference in the general hydration status, with polydipsic patients tending toward hyperhydration, while diabetes insipidus patients - who had to stop desmopressin for $24 \mathrm{~h}$ - might have been slightly dehydrated. Although patient numbers are too small to draw general conclusions, clinicians should give special caution about unexpected concentration differences in these populations.

Previous studies performed in the setting of acute illness have described the discrepancy between the indirect and direct measured sodium levels primarily to changes in protein and lipid concentrations $(8,9,10,11$, 12). Interestingly, in our cohort the discrepancy between the two measurement methods - showing systematically lower sodium measurements for the direct ISE method - increased during the course of the hypertonic saline infusion. For the indirect and direct ISE method, comparable results are expected if the sample properties are within the normal range, meaning plasma consists of approximately 93\% plasma water and 7\% lipid/protein $(13,14,15)$. A possible reason for the discrepant results could therefore be that hypertonic saline infusion leads to an osmotic change of the plasma-to-water ratio. This mechanism is supported by studies from the intensive care setting, where hypertonic saline was used as a 
resuscitation agent in sepsis or in raised intracranial pressure due to its transient volume expansion (26, 27). Accordingly, the direct ISE method would be more reliable in such situation. This finding is in line with other studies suggesting the use of the direct ISE method in patients where changes in plasma water are to be expected $(8,9,10)$.

Another pitfall in the interpretation of sodium levels is the s.D. of difference between different analyzers. As an example, if two analyzers show both a s.D. of difference of $1.2 \mathrm{mmol} / \mathrm{L}$, this could lead to a discrepancy of more than $4 \mathrm{mmol} / \mathrm{L}$ with the same patient material and a simultaneous measurement. It is therefore important for clinicians to be aware of the laboratory-specific measurement variances to prevent false diagnostic and therapeutic decisions when interpreting sodium levels (8).

Our study has some limitations. Firstly, sodium levels were measured with different devices due to the multicenter study design. Although correlation of the two measurement methods differed between the two main study centers, the tendency of the indirect ISE measurement resulting in higher sodium levels was consistent. Also, as the different measurement methods lead to the same conclusion, this supports the results of the data. Secondly, with 231 included participants, our cohort is smaller compared to other evaluations in this field. However, the repeated measuring during osmotic stimulus allowed to compare a total of 878 measurements by the two different ISE methods. Strengths of our study include that participants were recruited from two welldesigned prospective studies with strict inclusion criteria and that all participants were clinically stable without confounders such as acute illness. Importantly, with the controlled increase in plasma sodium values and repetitive simultaneous measurements, the course of the sodium differences could be systematically studied. It should be noted, however, that our results cannot be extrapolated to measurement discrepancies in the hyponatremic range. Nevertheless, considering the existing literature which showed similar differences in hyponatremic patients (9), a careful approach to the simultaneous use of the two measurement methods appears appropriate in hyponatremic patients.

In conclusion, we here show that intra-individual sodium levels differ significantly between the indirect and direct ISE measurement method and that this difference increases in the hypernatremic range. It is therefore crucial to adhere to the same measurement method in critical situations to avoid false decisions due to measurement differences.

\section{Supplementary data}

This is linked to the online version of the paper at https://doi.org/10.1530/ EJE-19-0101.

\section{Declaration of interest}

Martin Fassnacht is an associate editor for the European Journal of Endocrinology. He was not involved in the editorial or review process for this paper, on which he is listed as an author. The other authors have nothing to disclose.

\section{Funding}

The study was investigator initiated and was supported by a grant from the Swiss National Science Foundation to M Christ-Crain (SNF-162608) and the University Hospital Basel, Switzerland. W Fenske was supported by the Federal Ministry of Education and Research (BMBF) Germany (FKZ: 01EO1501) and Deutsche Forschungsgemeinschaft (DFG) Germany (AOBJ: 624808).

\section{Acknowledgements}

The authors thank all participants for their valuable contribution to our study. We thank the clinical staff, especially Cemile Bathelt, Nina Hutter and Anna Schrader for their valuable help during the study conduction.

\section{References}

1 Adrogué HJ \& Madias NE. Hyponatremia. New England Journal of Medicine 2000342 1581-1589. (https://doi.org/10.1056/ NEJM200005253422107)

2 Upadhyay A, Jaber BL \& Madias NE. Incidence and prevalence of hyponatremia. American Journal of Medicine 2006119 (7 Supplement 1) S30-S35. (https://doi.org/10.1016/j.amjmed.2006.05.005)

3 Adrogué HJ. Consequences of inadequate management of hyponatremia. American Journal of Nephrology 200525 240-249. (https://doi.org/10.1159/000086019)

4 Greenberg A, Verbalis JG, Amin AN, Burst VR, Chiodo JA, Chiong JR, Dasta JF, Friend KE, Hauptman PJ, Peri A et al. Current treatment practice and outcomes. Report of the hyponatremia registry. Kidney International 201588 167-177. (https://doi.org/10.1038/ki.2015.4)

5 Bataille S, Baralla C, Torro D, Buffat C, Berland Y, Alazia M, Loundou A, Michelet P \& Vacher-Coponat H. Undercorrection of hypernatremia is frequent and associated with mortality. BMC Nephrology 201415 37. (https://doi.org/10.1186/1471-2369-15-37)

6 Külpmann WR. Reference methods for the determination of sodium, potassium, $\mathrm{pH}$ and blood gases with ion-selective electrodes. European Journal of Clinical Chemistry and Clinical Biochemistry 1991 29 263-267. (https://doi.org/10.1515/cclm.1991.29.4.263)

7 Levy GB. Determination of sodium with ion-selective electrodes. Clinical Chemistry 198127 1435-1438.

8 Burnett RW, Covington AK, Fogh-Andersen N, Külpmann WR, Lewenstam A, Maas AH, Müller-Plathe O, Sachs C, SiggaardAndersen O, VanKessel AL et al. Recommendations for measurement of and conventions for reporting sodium and potassium by ionselective electrodes in undiluted serum, plasma or whole blood. International Federation of Clinical Chemistry and Laboratory Medicine (IFCC). IFCC Scientific Division Working Group on Selective Electrodes. Clinical Chemistry and Laboratory Medicine 2000 38 1065-1071. (https://doi.org/10.1515/CCLM.2000.159)

9 Solak Y. Comparison of serum sodium levels measured by blood gas analyzer and biochemistry AutoAnalyzer in patients with hyponatremia, eunatremia, and hypernatremia. American Journal of 
Emergency Medicine 201634 1473-1479. (https://doi.org/10.1016/j. ajem.2016.04.037)

10 Dimeski G, Morgan TJ, Presneill JJ \& Venkatesh B. Disagreement between ion selective electrode direct and indirect sodium measurements: estimation of the problem in a tertiary referral hospital. Journal of Critical Care 201227 326.e9-326.e16. (https://doi. org/10.1016/j.jcrc.2011.11.003)

11 Langelaan MLP, Kamp L, Zandijk E \& Raijmakers MTM. Prevalence of pseudonatremia in a clinical laboratory - role of the water content. Clinical Chemistry and Laboratory Medicine 201755 546-553. (https:// doi.org/10.1515/cclm-2016-0401)

12 Milionis HJ, Liamis GL \& Elisaf MS. The hyponatremic patient: a systematic approach to laboratory diagnosis. Canadian Medical Association Journal 2002166 1056-1062.

13 Story DA, Morimatsu H, Egi M \& Bellomo R. The effect of albumin concentration on plasma sodium and chloride measurements in critically ill patients. Anesthesia and Analgesia 2007104 893-897. (available at: https://insights.ovid.com/crossr ef?an=00000539-200704000-00029). (https://doi.org/10.1213/01. ane.0000258015.87381.61)

14 Dimeski G \& Barnett RJ. Effects of total plasma protein concentration on plasma sodium, potassium and chloride measurements by an indirect ion-selective electrode measuring system. Critical Care and Resuscitation 20057 12-15.

15 Fortgens P \& Pillay TS. Pseudohyponatremia revisited: a modernday pitfall. Archives of Pathology and Laboratory Medicine 2011135 516-519. (https://doi.org/10.1043/2010-0018-RS.1)

16 Verbalis JG, Goldsmith SR, Greenberg A, Korzelius C, Schrier RW, Sterns RH \& Thompson CJ. Diagnosis, evaluation, and treatment of hyponatremia: expert panel recommendations. American Journal of Medicine 2013126 S1-S42. (https://doi.org/10.1016/j. amjmed.2013.07.006)

17 Fenske W, Refardt J, Chifu I, Schnyder I, Winzeler B, Drummond J, Ribeiro-Oliveira A, Drescher T, Bilz S, Vogt DR et al. A copeptinbased approach in the diagnosis of diabetes insipidus. New England Journal of Medicine 2018379 428-439. (https://doi.org/10.1056/ NEJMoa1803760)
18 Fenske WK, Schnyder I, Koch G, Walti C, Pfister M, Kopp P, Fassnacht M, Strauss K \& Christ-Crain M. Release and decay kinetics of copeptin vs AVP in response to osmotic alterations in healthy volunteers. Journal of Clinical Endocrinology and Metabolism 2018103 505-513. (https://doi.org/10.1210/jc.2017-01891)

19 R core Team. R: A Language and Environment for Statistical Computing, 2018 R Foundation for Statistical Computing, Vienna, Austria. (available at: https://www.r-project.org)

20 Spasovski G, Vanholder R, Allolio B, Annane D, Ball S, Bichet D, Decaux G, Fenske W, Hoorn EJ, Ichai C et al. Clinical practice guideline on diagnosis and treatment of hyponatraemia. European Journal of Endocrinology 2014170 G1-G47. (https://doi.org/10.1530/ EJE-13-1020)

21 Sterns RH \& Silver SM. Complications and management of hyponatremia. Current Opinion in Nephrology and Hypertension 2016 25 114-119. (https://doi.org/10.1097/MNH.0000000000000200)

22 Laureno R \& Karp BI. Pontine and extrapontine myelinolysis following rapid correction of hyponatraemia. Lancet $1988 \mathbf{1}$ 1439-1441. (https://doi.org/10.1016/S0140-6736(88)92247-7)

23 Liamis G, Milionis H \& Elisaf M. A review of drug-induced hyponatremia. American Journal of Kidney Diseases 200852 144-153. (https://doi.org/10.1053/j.ajkd.2008.03.004)

24 Stachenfeld NS, Keefe DL \& Taylor HS. Responses to a saline load in gonadotropin-releasing hormone antagonist-pretreated premenopausal women receiving progesterone or estradiolprogesterone therapy. Journal of Clinical Endocrinology and Metabolism 200590 386-394. (https://doi.org/10.1210/jc.2004-0941)

25 Stachenfeld NS \& Taylor HS. Sex hormone effects on body fluid and sodium regulation in women with and without exercise-associated hyponatremia. Journal of Applied Physiology 2009107 864-872. (https://doi.org/10.1152/japplphysiol.91211.2008)

26 Oliveira RP, Velasco I, Soriano FG \& Friedman G. Clinical review: hypertonic saline resuscitation in sepsis. Critical Care 20026 418-423.

27 Qureshi AI \& Suarez JI. Use of hypertonic saline solutions in treatment of cerebral edema and intracranial hypertension. Critical Care Medicine 200028 3301-3313. (https://doi. org/10.1097/00003246-200009000-00032)

Received 13 February 2019

Revised version received 10 May 2019

Accepted 4 June 2019 